\title{
FURTHER INEQUALITIES FOR SECTOR MATRICES
}

\author{
DENGPENG ZHANG* AND NING ZHANG
}

Abstract. We mainly generalize a norm inequality of $n \times n$ block accretive-dissipative matrices. This complements the results of Kittaneh [10, Theorem 2.4] and Fu [18, Theorem 2.9]. And then, we present some singular value inequalities for sector matrices.

Mathematics subject classification (2020): 15A60, 15A45, 15A18.

Keywords and phrases: Accretive-dissipative matrix, singular value, sector matrix, norm inequalities.

\section{REFERENCES}

[1] M. Alakhrass, A note on sectorial matrices, Linear Multilinear Algebra, available from, https://doi.org/10.1080/03081087.2019.1575332.

[2] R. Bhatia, Matrix Analysis, GTM 169, Springer-Verlag, New York (NY), 1997.

[3] R. Bhatia, J. A. R. Holbrook, On the Clarkson-McCarthy inequalities, Math Ann. 281, (1988), $7-12$.

[4] R. Bhatia, F. Kittaneh, Norm inequalities for positive operators, Lett Math Phys. 43, (1998), $225-231$.

[5] J. C. BOURIn, M. UCHIYAM, A matrix subadditivity inequality for $f(A+B)$ and $f(A)+f(B)$, Linear Algebra Appl. 423, (2007), 512-518.

[6] I. Garg, J. Aujla, Some singular value inequalities, Linear Multilinear Algebra 66, (2018), 776 784.

[7] I. H. Gumus, O. HirZallah, F. KitTAnEH, Norm inequalities involving accretive-dissipative $2 \times 2$ block matrices, Linear Algebra Appl. 528, (2017), 76-93.

[8] R. A. Horn And C. R. Johnson, Topics in Matrix Analysis, Cambridge University Press, Cambridge, 1991.

[9] L. Hou, D. P. ZHANG, Concave functions of partitioned matrices with numerical ranges in a sector, Math Inequal Appl. 20, (2017), 83-589.

[10] F. KitTANeh, M. SAKKIJHA, Inequalities for accretive-dissipative matrices, Linear Multilinear Algebra 67, (2019), 1037-1042.

[11] E. Y. LEE, Extension of Rotfel'd theorem, Linear Algebra Appl. 435, (2011), 735-741.

[12] C. K. Li, R. Mathias, Generalizations of Ky Fans dominance theorem, SIAM J. Matrix Anal. Appl. 19, (1998), 99-106.

[13] C. K. LI, S. N. SZE, Determinantal and eigenvalue inequalities for matrices with numerical ranges in a sector, J. Math. Anal. Appl. 410, (2014), 487-491.

[14] M. LIN, Some inequalities for sector matrices, Oper. Matrices 10, (2016), 915-921.

[15] M. LIN, Fischer type determinantal inequalities for accretive-dissipative matrices, Linear Algebra Appl. 438, (2013), 2808-2812.

[16] M. LiN, F. SUN, A property of the geometric mean of accretive operator, Linear Multilinear Algebra 65, (2017), 433-437.

[17] M. Lin, D. ZHOU, Norm inequalities for accretive-dissipative operator matrices, J. Math. Anal. Appl. 407, (2013), 436-442.

[18] S. Lin, X. FU, On some inequalities for sector matrices, Linear Multilinear Algebra, available from, https://doi.org/10.1080/03081087.2019.1600466.

[19] Y. MAO, X. LiU, On some inequalities for accretive-dissipative matrices, Linear Multilinear Algebra, available from, https://doi.org/10.1080/03081087.2019.1635566. 
[20] L. NASIRI, S. FURUICHI, New inequalities for sector matrices applying Garg-Aujla inequalities, available from, https://arxiv.org/abs/2001.00687.

[21] C. YAnG, F. LU, Some generalizations of inequalities for sector matrices, J. Inequal. Appl. 2018, (2018), 183.

[22] D. Zhang, L. Hou, L. MA, Properties of matrices with numerical ranges in a sector, Bull. Iranian Math. Soc. 43, (2017), 1699-1707.

[23] F. ZhANG, A matrix decomposition and its application, Linear Multilinear Algebra 63, (2015), 20332042.

[24] F. Zhang, Matrix Theory: Basic Results and Techniques, Universitext, Springer, New York, 1999. 